\title{
LAS LIGADURAS DE ULISES O LA SUPUESTA NEUTRALIDAD VALORATIVA DE LA CIENCIA Y LA TECNOLOGÍA
}

\author{
Eulalia Pérez Sedeño \\ Dep. de Ciencia, Tecnologia y Sociedad. Instituto de Filosofía. CSIC
}

\begin{abstract}
According to classic philosophy of science, science is impartial, autonomous and value free. In the base of this conception is a distinction between objective values and subjective values, also denominated cognitive or constituent values and non-cognitive or contextual values. In this paper some critics to the value-free idea of science, their autonomy and impartiality are reviewed; in special it is shown the relevant role that all kind of values carry out in science, and the importance of 'gender' category in performing these analyses, as the different papers in this issue show.
\end{abstract}

KEY WORDS Fact and values. Constitutive and contextual values. Gender. Kuhn.
RESUMEN Según la filosofía clásica de la ciencia, la ciencia es imparcial, autónoma y valorativamente neutra. Al afirmar esto último, se está distinguiendo entre valores objetivos y valores subjetivos, también denominados valores cognitivos o constitutivos y valores no cognitivos o contextuales. En este artículo se repasan algunas criticas a la neutralidad valorativa de la ciencia, su autonomía e imparcialidad; en especial se muestra el relevante papel que los valores de todo tipo desempeñan en la ciencia, asi como la importancia de la categoría género en estos análisis, tal y como muestran los diversos ensayos que aparecen en este número.

PALABRAS CLAVE Hechos y valores. Valores constitutivos y contextuales. Género. Kuhn.
La filosofía de la ciencia clásica, heredera del positivismo lógico y del Círculo de Viena, también conocida como la "concepción heredada" o "la Leyenda" ${ }^{1}$, mantiene que la ciencia se ocupa de los hechos, no de valores, y que los primeros son objetivos, mientras que los segundos no. Aunque esta distinción es compleja, podemos decir que enfrenta cuestiones de hecho versus cuestiones de valor 0 , dicho de otro modo, enunciados sobre lo que es, frente a oraciones sobre lo que debe ser. Los primeros, los enunciados fácticos, hacen referencia a estados de hechos y pueden ser verdaderos o falsos, mientras en el segundo caso nos encontramos con oraciones normativas o evaluativas que adscriben a un agente la obligación de actuar, o atribuyen la cualidad de bueno a algo. La ciencia contiene, sobre todo, enunciados de hechos, por lo que, esa reflexión de segundo orden que es la filosofía de la ciencia, sólo se ocuparía de ese tipo de enunciados, acerca de "lo que es», quedando los juicios de valor, sobre "lo que debe ser", relegados a otro dominio. A la separación dicotómica entre hechos y valores se unian otras distinciones como contexto de descubrimiento/contexto de justificación o entre ciencia pura/aplicación de la ciencia que avalaban la idea de una ciencia (y una persona de ciencia) pura y valorativamente neutra, en la que consideracio- nes éticas, sociales, etc. no tenían cabida. En el contexto de justificación, el único que es competencia exclusiva de la filosofía de la ciencia, sólo consideraciones lógico-empíricas eran tenidas en cuenta; según la otra distinción, no hay ciencia buena o mala, sino que son las personas que la utilizan la que hacen buen o mal uso de ella.

A esta distinción entre hechos y valores subyace una concepción que considera que la ciencia es imparcial, autónoma y neutral. Según esta concepción la imparcialidad de la ciencia significa que nuestras creencias científicas están determinadas o avaladas por los hechos o por criterios imparciales o no arbitrarios de decisión acerca de ellas, y no por nuestros deseos de cómo deben ser las cosas: en cualquier caso, jamás por valores pertenecientes al contexto sociocultural; también esa concepción considera que la ciencia es autónoma, es decir, que progresa mejor cuando no está influida por intereses, valores o movimientos politicos, sociales o del tipo que fuere; y que es neutral, esto es, que nuestras teorias no implican ni presuponen juicio alguno acerca de valores no cognitivos y que las teorias científicas tampoco sirven más a unos valores contextuales concretos que a otros ${ }^{2}$. 
Cuando se afirma que la ciencia está libre de valores o la neutralidad valorativa de la ciencia, se está haciendo una distinción, implícita o explícitamente, entre valores objetivos y valores subjetivos, también denominados valores cognitivos 0 constitutivos y valores no cognitivos o contextuales. Se considera que los primeros son propios de la ciencia y suelen mencionarse como tales la verdad o la verdad aproximada, la precisión predictiva, la adecuación empírica, el poder explicativo, la simplicidad o la elegancia. Las normas, preferencias, creencias e intereses que no tienen que ver con los valores cognitivos se denominan "contextuales" porque varían según el contexto científico y suelen ser de tipo ideológico, económico, religioso, etc.: si se eligiera, por ejemplo, una hipótesis o teoría por la raza o el género de quien la propone, diriamos que esos valores contextuales están influyendo en la elección de hipótesis o teorias. Aunque este tipo de influjo puede parecer extraño en principio, hay otros casos en que no lo parece tanto: por ejemplo, cuando la institución científica a la que pertenece quien propone una hipótesis o teoría o efectúa determinado experimento influye decisivamente en su aceptación. En realidad, se suele admitir que cualquier persona que se dedica a la ciencia tiene, y por tanto se ve influida por, valores no epistémicos, como la honestidad, la lealtad a los colegas, la aceptación de sus pares, etc. Pero la tesis de la neutralidad valorativa de la ciencia es algo más fuerte. Lo que afirma es que, cuando los valores contextuales se encuentran presentes en el proceso de evaluación y aceptación de teorias, se está haciendo mala ciencia; por tanto, los valores contextuales son perniciosos para la buena ciencia.

Las críticas a la neutralidad valorativa de la ciencia, su autonomía e imparcialidad y en especial al papel que los valores de todo tipo desempeñan en ella han sido y son diversas. Pero, si nos atenemos a los textos que más influjo han tenido al respecto, tendremos que responsabilizar de ello a T. S. Kuhn $(1962,1977)$. En La Estructura de la Revoluciones Científicas, Kuhn introdujo el término «paradigma'y, ante las acusaciones (entre otras) de la vaguedad que conlleva el término ${ }^{3}$, en el Postescripto añadido unos años después decidió sustituirlo por el de umatriz disciplinar»: "Tal y como se utiliza usualmente en filosofía de la ciencia... "teoría" connota una estructura mucho más limitada en su naturaleza y alcance que lo que necesitamos aqui. Hasta que pueda ser liberado el término de sus implicaciones actuales, evitaremos confusiones si adoptamos otro. Para mis propósitos actuales sugiero «matriz disciplinarn: "disciplinar» porque refiere a lo que poseen en común quienes practican una determinada disciplina; "matriz», porque se compone de elementos ordenados de diversos tipos, cada uno de los cuales exige especificaciones adicionales" ${ }^{4}$. Los cuatro componentes son las generalizaciones simbólicas (que se corresponden bastante con las leyes 0 teorias en sentido tradicional), los supuestos metafísicos ${ }^{5}$, los ejemplares ${ }^{6}$ y los valores: "Probablemente, los valores que se mantienen más profundamente tienen que ver con las predicciones: deben ser precisas; las predicciones cuantitativas son preferibles a las cualitativas; sea cual fuere el margen de error permisible, debe ser consistentemente satisfecho en un campo dado, etc. Sin embargo, también hay valores que se usan para juzgar todas las teorias: deben, en primer lugar y por encima de todo, permitir la formulación y resolución de problemas; siempre que sea posible, deben ser simples, autoconsistentes y plausibles, compatibles, esto es, con otras teorías mantenidas en la actualidad... También existen otro tipo de valores -por ejemplo deben (o no necesitan) ser socialmente útilespero lo anterior indica lo que tengo en la cabezan? 7 .

No obstante, seguramente fue su artículo de $1977^{8}$ el que avivó más la polémica sobre la tesis de que la ciencia es (y debe ser) un proceso en el que interactúan tanto valores cognitivos como no cognitivos. En realidad, en este trabajo Kuhn intenta responder a quienes le han acusado de relativista e irracionalista por las afirmaciones que hiciera en $L a$ Estructura acerca de la resolución de los problemas de decisión entre teorias alternativas. Al preguntarse por las características de una buena teoría, y en línea con lo expuesto anteriormente selecciona cinco -precisión o adecuación empírica, coherencia (por supuesto interna, pero, sobre todo, externa), amplitud o alcance, simplicidad y fecundidad- que "constituyen la base compartida para la elección de teorias ${ }^{9}$, junto con otras. La novedad del tratamiento reside en que Kuhn afirma que, cuando los individuos aplican estos valores a casos concretos, los individuos pueden discrepar, lo cual evidentemente indica que no son unívocos, ni generales y que hay que tomar en cuenta el sujeto que los aplica.

Examinemos brevemente estos valores. La precisión o adecuación empírica es el valor más determinante porque es el menos equivoco y por su carácter predictivo-explicativo; dicha precisión puede ser cualitativa o cuantitativa. Pero, como el propio Kuhn señala, la importancia y el carácter determinativo de este valor no está siempre claro: en el caso de la controversia entre las teorias del oxígeno y del flogisto, la primera era más precisa en su explicación de las relaciones de peso en ciertas reacciones químicas, pero la del 
flogisto precisaba y explicaba por qué los metales eran más semejantes entre sí que en relación con los minerales de los que provenian. En el caso de la astronomía ptolemaica y la copernicana, en el momento en que esta última fuera formulada por Copérnico, la primera era más precisa y daba cuenta de más hechos que la segunda. ¿Por qué se impuso ésta? La conclusión a la que llega Kuhn es que la precisión sola no basta, son necesarios otros valores (aunque, como veremos, no eliminan los problemas). ¿Qué sucede cuando los otros valores mencionados entran en juego?

Tanto la teoría astronómica ptolemaica como la copernicana eran internamente coherentes. No obstante, la ptolemaica era coherente externamente con la física aristotélica (es decir, compatible con otra teoría existente, lo que no sucedia con su oponente). $Y$, por lo que se refiere a la simplicidad, fue un valor que favoreció a la teoria copernicana, pero "evaluada de forma especial»: por lo que se refiere a cálculos técnicos, ambas eran igualmente simples (o complejas), pero la teoría copernicana ganaba en cuanto a características generales, pues sólo exigía un círculo para cada planeta, en vez de dos, tres o cuatro necesarios según el astro, en la ptolemaica.

Asi pues, según Kuhn cuando hay que elegir entre dos teorias alternativas, dos personas que compartan los mismos valores o criterios generales pueden llegar a conclusiones distintas. Si queremos averiguar por qué, tendremos que "trascender la lista de criterios compartidos y pasar a las caracteristicas de los individuos que tomaron las decisiones. Esto es, deben tratarse características que varian de un científico a otro, sin que, por ello, se ponga en peligro su apego a los cánones que hacen que la ciencia sea científica" ${ }^{10}$. Esas diferencias tendrán que ver con "las experiencias del científico como individuo", "por ejemplo, en qué parte trabaja, qué parte de su trabajo depende de los conceptos o hipótesis impugnados por el nuevo paradigma, con valores externos a la propia ciencia ${ }^{11}$, o con factores peculiares de la personalidad del propio científico.

Naturalmente, estos factores son precisamente del tipo subjetivo impugnado por la Leyenda, pues, afirman los criticos de Kuhn, pertenecen al denominado contexto de descubrimiento $y$, si bien los valores subjetivos pueden desempeñar cierto papel en dicho contexto, no tienen cabida en lo que se considera competencia de la filosofia de la ciencia, el contexto de justificación, el ámbito de la objetividad. Pero, como señala Kuhn, aunque la distinción entre ambos contextos pueda ser pedagógicamente interesante -esto es, en la "ciencia del libro de texto"- no responde al funcionamiento real de la ciencia en lo que respecta a la toma de decisiones.

Por ejemplo, cuando se enseña una teoría, se utilizan una o varias aplicaciones ejemplares que, a la vez, son consideradas prueba de la teoría ${ }^{12}$. Por otro lado, los denominados "experimentos cruciales» se presentan como casos paradigmáticos de «buenas razones para la elección científica, ilustran la más eficaz de todas las clases de argumentos que tiene a su alcance el científico cuando no sabe cuál de entre dos teorias elegir y son los vehículos para la transmisión de los criterios de elecciónı13; pero dichos experimentos cruciales tienen la caracteristica adicional de que, cuando se realizaron, los científicos ya tenían tomada la decisión y sólo son pertinentes cuando producen resultados inesperados. Resumiendo, los filósofos de la ciencia sólo analizan los argumentos favorables a la teoria que finalmente triunfó, eliminando un elemento esencial en las situaciones de toma de decisiones: siempre hay buenas razones a favor y en contra de una hipótesis o teoria, en el momento en que se toman las decisiones personalmente (subjetivamente) por lo que "las consideraciones pertinentes al contexto de descubrimiento son, pues, pertinentes también al contexto de justificación» ${ }^{14}$.

Para Kuhn, al igual que para muchos posteriormente, no es posible dudar de la existencia en todos los niveles de "valores subjetivos». Pero ¿es eso pernicioso para la ciencia? No sólo no lo es, señala Kuhn, sino que, además, el hecho de que sean necesarios, además de los "objetivos" para llevar a cabo la elección, es una ventaja, no una desventaja.

Kuhn distingue entre máximas, reglas y valores. Las primeras, que suelen ser vagas y entran en conflicto a menudo, tomadas una a una prescriben elecciones diferentes y consideradas conjuntamente no prescriben ninguna. Los valores y normas, constituyen una guía más eficaz ante conflictos y errores, pues proporcionan ejemplos claros para ello y pueden variar históricamente. Por ejemplo, al valor de «mejorar la vida» estuvo asociada durante varios años la norma "una casa, un cochen. Pero al tener en cuenta otros aspectos de la calidad de vida que atañen a otros valores (la destrucción de la capa de ozono o el agotamiento y encarecimiento de los recursos petroliferos) la norma varía ${ }^{15}$. Dicho de otro modo, los criterios que enumera, aunque sean ambiguos, especifican "lo que cada científico 
debe tomar en cuenta para llegar a una decisión, lo que puede considerar pertinente o no y lo que puede pedirsele legitimamente que comunique como base de la elección tomada", funcionando, pues, "no como reglas que determinan decisiones a tomar, sino como valores que influyen en éstas» ${ }^{16}$.

La empresa científica siempre se realiza en un contexto cultural concreto, por lo que las personas de ciencia siempre e inevitablemente incorporarán valores de su propia cultura en la práctica científica, sea consciente o inconscientemente. Eso no tiene que ser necesariamente pernicioso, pues, como veremos, algunos valores nos dicen cómo manejar el sesgo potencial que pueden introducir otros valores, de modo que podamos conseguir un conocimiento más fiable. De hecho, cuanta mayor diversidad de valores intervengan, podremos obtener mejor conocimiento allá donde se produzca su intersección. Por otro lado, los valores contextuales no tienen por qué ser subjetivos, entendiendo por ello no razonados ni justificados: si esos valores se adoptan basándose en principios generales, discutidos y justificados comunitariamente, pueden ser tan objetivos como los epistémicos, es decir, intersubjetivos.

En efecto, es posible que de los valores últimos de la ciencia se deriven otros más próximos o inmediatos. Detengámonos por un momento en el ethos mertoniano encarnado en el conocido mecanismo de los CUDEOS $^{17}$ : normas, valores o "imperativos institucionales» que contribuyen al crecimiento del conocimiento público. Estos incluyen el Comunitarismo (esto es, el conocimiento debe ser un bien común, comunicado a toda la comunidad), el Universalismo (los criterios de evaluación han de ser universales, sin que influyan en ellos factores tales como la raza, la clase, el género o cualquier otra característica social o personal de los investigadores), el Desinterés (los investigadores deben ser humildes en diversos sentidos del términos y no deben estar guiados por intereses que no sean la obtención de conocimiento) y, finalmente el Escepticismo Organizado (la autoridad no debe sesgar ni guiar el conocimiento -todos los investigadores están investidos de igual autoridad, todos son "pares»- y todo conocimiento es, en principio, provisional y puede, tarde o temprano, resultar falso). Aunque como el propio Merton ya indicó, estas normas no siempre se cumplen ${ }^{18}$, ayudan, sin embargo, a establecer ciertas condiciones básicas o ciertos valores que contribuyen a que una comunidad acepte como conocimiento certificado cierto tipo y no otro $^{19}$

ARBOR CLXXXI 716 NOVIEMBRE-DICIEMBRE (2005) 447-462 ISSN: 0210-1963
Tanto las normas institucionales como otros valores epistémicos son elementos del proceso importantísimos en la ciencia, pero, son contingentes o contextuales: Tal es el caso de la evaluación de las afirmaciones de conocimiento, lo que incluye la observación y experimentación controlada, la confirmación de predicciones, la repetitividad de las experiencias $y$, muchas veces, el análisis estadístico. Pero, al margen de que no todos esos procedimientos son factibles en todas las disciplinas o subdisciplinas, estos valores son históricos, es decir, han variado, varian, a lo largo de la historia y es nuestra experiencia a lo largo de ella la que los conforma. Por indicación de Lady Montagu y a instancias del Colegio de Médicos de Londres se inoculó la viruela a seis reos -a cambio de lo cual se les conmutó la pena de muerte- y luego a otros tantos huérfanos; a todos ellos se les puso en contacto con enfermos para ver si la inoculación funcionaba y no enfermaron. Eso plantea una cuestión que nos llevaría muy lejos, pero que no podemos dejar de mencionar aqui porque resulta fundamental: la necesidad de introducir criterios contextuales, y no sólo epistémicos, a la hora de seleccionar o evaluar hipótesis o teorias, para evitar, de ese modo, prácticas científico-tecnológicas que perjudican de forma clara a ciertos grupos o personas, sean éstos mujeres, homosexuales, judios o negros. Pero lo cierto es que la vieja concepción de "la Leyenda" excluia -aún pretende excluir- la posibilidad de que eso suceda en los casos de "buena ciencia». Tal ha sido la tradición que ha posibilitado, en aras de una supuesta "buena cientificidad" el desarrollo de teorias racistas, sexistas, etc. y el uso de prácticas pasadas y presentes reprobables desde un punto de vista ético: desde el modo en que Jenner ideó y probó la vacuna de la viruela, los ensayos de Semmelweiss para descubrir el agente causante de la fiebre puerperal en el Hospital General de Viena, los experimentos con judios y gitanos de ciertos médicos alemanes antes y durante la Segunda Guerra Mundial para averiguar el tiempo de resistencia de los cuerpos humanos en condiciones de hipotermia, hasta recientes experiencias con fármacos como las pildoras anticonceptivas o cócteles anti-S.I.D.A.

Y ni siquiera los experimentos aparentemente neutrales están libres de críticas, pues subyacen a ellos una serie de supuestos y valores a veces no suficientemente tenidos en cuenta. Por ejemplo, la pertinencia de extrapolar de otras especies animales a la humana ha sido cuestionada también en el caso de la medicina y la biología, como ha sucedido recientemente con el fármaco experimental TGN 1412, fabricado por la compañía alemana TeGenero. Dicho 
fármaco es una proteína desarrollada genéticamente para abordar la proteina $\mathrm{CD} 28$, presente en las células T que son una clase de leucocitos. EI TGN 1412 está diseñado para activar una molécula situada en la superficie de los leucocitos, la cual desencadena, a su vez, una reacción química que se supone ayuda a los glóbulos a reconocer y destruir ciertas células relacionadas con la leucemia y la esclerosis en placas. Pues bien, después de ensayar dicho fármaco con ratones, conejos y perros fue inyectado en 6 humanos en una dosis 500 veces menor a la usada con otros animales, sufriendo un fallo multisistémico que los mantiene en estado crítico (dos de ellos en coma).Aparte de una serie de cuestiones importantes que este ensayo ha suscitado en la comunidad médica (y que debería plantearse a nivel general en la sociedad) diversos científicos han manifestado que quizás "la droga funciona de manera distinta en los animales que en los hombres [sic] y que, por eso, los animales usados en los ensayos previos no sufrieron las mismas reacciones, porque los anticuerpos fabricados para el TGN 1412 estaban diseñados específicamente para afectar a la proteina humana» 20 .

Los valores contextuales, sean del tipo que fueren, pueden contribuir, y de hecho lo hacen, a conformar los valores epistémicos, asi como a constreñirlos: incluso, pasándonos al terreno normativo, podriamos decir que deben hacerlo. Además, en muchas ocasiones los valores cognitivos han ocultado u ocultan otros valores éticos o sociales que no conviene que desaparezcan y que en muchos casos llegan a formar una parte importante del proceder científico. No sólo por lo que respecta a valores éticos de los mencionados en los casos anteriores, sino que, constantemente se exige que se de un tratamiento adecuado a los sujetos experimentales, precisando y variando nuestra noción de "tratamiento adecuado" a lo largo de la historia, que en estos momentos alcanza también a animales no humanos, hasta el punto de que, en muchas ocasiones, se debata si es adecuado utilizar los resultados obtenidos con pruebas experimentales "empiricamente fiables" pero "éticamente indeseablesı ${ }^{21}$. Pero, además es que algunos de esos valores éticos son auténticamente "constitutivos" de la práctica científica, como la honestidad y la honradez: se supone que los investigadores informarán de los resultados obtenidos realmente, que no engañarán, ni ocultarán ninguna información relevante. Es cierto que en muchas ocasiones estos valores se transgreden, pero, precisamente el desafío que supone lograr un equilibrio entre los ideales y la realidad, ayuda a mejorar nuestra ciencia. Infringir esas normas suele pasar factura, como bien saben, por ejemplo, el coreano Woo-suk Hwang, que alteró parte de sus resultados en clonación, o el chino Chen Ji, quien en 2003 presentó como propios microprocesadores conseguidos en una empresa extranjera ${ }^{22}$. ¿Qué es si no un intento de proteger e introducir valores sociales en la ciencia, los protocolos de investigación en seres humanos, la utilización de animales no humanos en el laboratorio o las normas para perseguir y castigar el fraude?

Los valores sociales o éticos también intervienen en los temas o fines de la investigación. Aunque se ejecute siguiendo todas las normas de contrastación y experimentación, etc., no podemos decir que la investigación sobre armas mecánicas, nucleares o bacteriológicas, sea valorativamente neutral - como tampoco lo es la investigación sobre energía solar o sobre métodos agrícolas para obtener mejores cosechas. En cada uno de estos casos, no sólo «el investigador es un agente ético responsable de las consecuencias de sus acciones, sean buenas o malas " ${ }^{23}$, sino que hay una responsabilidad común que también atañe a la política científica. Algunos científicos y filósofos apelan a la distinción tradicional entre ciencia o investigación "pura" y "aplicada", pero eso no sirve de nada. Por un lado, porque en esta época de megaciencia (o big science) que nos toca vivir, resulta sumamente dificil separar la una de la otra ${ }^{24}$. Pero, sobre todo, porque cuando surgen los debates sobre la investigación - por ejemplo, en la investigación con embriones humanos ${ }^{25}$ - lo que surgen son auténticos conflictos entre valores éticos o sociales que la ciencia sola no puede resolver, ni tampoco puede considerar irrelevantes. Incluso algunos cientificos se dan cuenta de los valores presentes en su investigación "básica" y cómo la conforman políticamente ${ }^{26}$.

La retórica utilizada en la presentación de los resultados cientificos, del "conocimiento autorizado", a veces nos hace olvidar que la ciencia la efectúan seres humanos individuales, de carne y hueso, que poseen una serie de valores, los de la propia cultura en la que viven, además de los de la comunidad cientifica a la que pertenecen. $E$ incluso aunque uno de los principales valores (cognitivos) de la ciencia es el desarrollo (o la obtención) de conocimiento del mundo que nos rodea (adecuación o precisión empirica), esta obtención de conocimiento no se hace aisladamente, encerrados en torres de marfil, sino, por ejemplo, en paises concretos. Y la investigación -sea en medicina, astrofísica, mecánica cuántica o ciencias espaciales- no es

ARBOR CLXXXI 716 NOVIEMBRE-DICIEMBRE (2005) 447-462 ISSN: 0210-1963 
gratis. Asi pues, es probable -casi seguro- que haya que considerar ese valor epistémico en el contexto de otros valores.

Del mismo modo, los valores contextuales también pueden afectar la descripción de los datos, y los conceptos, esto es, se pueden utilizar términos cargados de valores a la hora de describir observaciones y experimentos y los valores pueden influir en la selección de los datos o en los tipos de fenómenos a investigar. Este tipo de influjo se ve muy bien en los casos de usos de ciertas metáforas pero, sobre todo, en disciplinas como la primatología o en teorías como la Sintesis Evolutiva.

Un ejemplo típico es el de Lucy. Cuando se descubrió en Etiopia un homínido fósil de 3,2 millones de años se lo llamó "Lucy" (por la canción de los Beatles "Lucy in the sky with diamonds"). "Lucy" es un nombre de mujer $y$, por tanto, el nombre dado implicaba que el fósil encontrado era el de una hembra. Ahora bien, por lo general, el sexo de los individuos se determina por los genitales o por el ADN. Como los primeros no se fosilizan y el segundo raras veces se encuentra en los fósiles, la determinación del sexo en el caso de los fósiles se hace, o bien por la morfología pélvica, por el tamaño corporal, o por los dientes caninos en los no humanos. Sólo por su tamaño se decidió que era uhembra", pues se supone que es inferior en las hembras que en los machos. Pero tal vez era un joven, o un macho pequeño de una especie donde habia machos grandes y pequeños o un macho de una especie en que todos los individuos eran pequeños ${ }^{27}$. Este tipo de suposiciones sesgadas también aparecen en muchas exposiciones o museos de ciencias naturales, donde el macho aparece mucho más grande que su pareja (a la que, además, suele abrazar protectoramente). Incluso en investigaciones supuestamente menos propensas a la inclusión de valores, también sucede. Por ejemplo, en la investigación agrícola, conceptos como sostenibilidad, precaución, bienestar animal, fertilidad del suelo o calidad de la naturaleza, están cargados de valores: según los usen diferentes grupos y en discursos distintos, tendrán diferentes significados.

También hay valores que pasan de la ciencia a la sociedad: la ciencia y la tecnología tienen éxito y son potentes, poderosas, las cosas que se asocian a ellas tienen más valor y autoridad y lo más dramático de todo es que se puede considerar que, puesto que se tiene la imagen de la ciencia como actividad resolutoria de problemas, es un modelo para la resolución de todos los problemas, científicos o no: se puede tender a considerar que la ciencia (y la tecnología) pueden solucionar todos los problemas sociales (como positivistas ingenuos) y que es el principal medio para lograr la objetividad. Pero, ¿qué sucede cuando hay otros valores implicados? El caso es que no todos los problemas son susceptibles de tener una solución científica. Por ejemplo, una visión estrecha nos llevaría a pensar que el problema poblacional tiene una solución tecnológica: si no hay suficientes recursos alimenticios para alimentar a toda la población del planeta, bastará habilitar las soluciones científico-tecnológicas adecuadas (tecnologías de control reproductivo, tecnologias que aumenten la eficacia agricola, etc.) Ahora bien, el problema conlleva también un reto ético acerca de la libertad de tener o no hijos en un mundo con recursos globales limitados, que no evitará la "tragedia de los comunes». Es decir, hay un tipo de problemas humanos que son "problemas que no tienen solución técnica» 28 , pues están implicadas al menos dos contradictorias. Por eso, lo que hay que lograr es un consenso acerca de cómo usar individualmente los recursos comunitarios.

Por supuesto que la ciencia puede ayudar a identificar relaciones causales o consecuencias imprevistas en las que los valores éticos, o de otro tipo, son importantes o relevantes. Por otro lado, para poder tomar decisiones necesitamos disponer de un buen conocimiento previo. El caso de evaluaciones de riesgo son típicas: los científicos pueden decir dónde, por qué, cómo y en qué medida existe un riesgo, pero si debemos evaluar en qué medida es uaceptable" un riesgo, es muy probable que necesitemos echar mano de otros valores. Es posible que, por ejemplo en casos de evaluación de riesgos, trasladar los valores "estrictamente científicos", como por ejemplo el ideal de cuantificación, oculte las dimensiones éticas y sociales relevantes: es posible que no baste con reducir el problema a una escala numérica, sino que haya que plantearse cuestiones acerca de la distribución del riesgo entre distintas personas o grupos, la autonomía de aquellas para aceptarlo, etc. Por eso es sumamente importante disponer de una buena educación en ciencia y tecnologia, a todos los niveles, de modo que personas informadas, aunque no expertas puedan tomar decisiones propias o participar en la toma de decisiones que afectan a su grupo, a la sociedad o a la humanidad, convirtiéndose esa participación en un elemento más, e importante, de la ciencia y la tecnologia. Lo contrario sería extender la ciencia más allá de su propio dominio, a la vez que la renuncia de la ciudadanía a sus propias responsabilidades. 
El conocimiento cientifico y las tecnologias, también pueden plantear nuevos problemas éticos o sociales como sucede en la hemodiálisis, los trasplantes de órganos y tejidos, la reproducción asistida o la ecología. Las nuevas situaciones "científicotecnológicas" nos obligan muchas veces a aplicar viejos valores de una nueva forma o a replantearnos los significados de viejos conceptos: por ejemplo, el de "vida/muerte" en tecnologías médicas, los de "padre", "madre" o "familia" en las tecnologías de reproducción asistida. Puede incluso que, cuando la ciencia y la tecnologia resuelven ciertos problemas, introducen nuevos problemas sobre valores que no pueden resolver, pero no por ello hay que excluirlos del dominio de la ciencia.

Cuando se defiende la autonomía y la neutralidad valorativas de la ciencia, parece darse por sentado que los demás valores que rigen la sociedad no necesitan justificación. Pero aunque puede que eso suceda en ciertas sociedades no democráticas ${ }^{29}$, no debe ocurrir asi en las sociedades democráticas: nuestros valores contextuales deben ser sometidos públicamente a crítica y justificados mediante cuidadosos razonamientos, apelando a evidencias $y$, seguramente, también, a sentimientos comunes compartidos, igual que sucede con los argumentos científicos. Del mismo modo que sucede en ciencia, tendremos que poder experimentar las consecuencias de nuestras acciones y universalizar nuestras decisiones. Estamos ligados por nuestros valores sociales, como Ulises al mástil de su barco y, si bien no podemos cortar las ligaduras, tal vez sea razonable para todos elegir con qué nos atamos. Ese es uno de los principales objetivos de los artículos que aparecen en este número.

Introducir en nuestros análisis el "género" (claramente contextual) y una perspectiva feminista arroja nueva luz sobre los problemas. Entendemos por género una categoría cultural que se opone a la de sexo. Por sexo entendemos las características "biofisicas" que diferencian a los hombres de las mujeres. No obstante, el sexo puede ser de diversos tipos: cromosómico, genital, gonadal, hormonal y se pueden dar combinaciones de al menos dos de los tipos de sexos anteriores. En cambio, el término "género" refiere de manera general a pautas de comportamiento, social y culturalmente especificas, ya sean reales o normativas y que tiene diversas dimensiones, tales como roles, normas, identidad, virtudes o simbolismo de género ${ }^{30}$. El concepto de género procede de la psicología y medicina clínica. John Money (1955) introdujo en la literatura psicológica el con- cepto rol de género -la expresión pública de ser varón o mujer- ${ }^{31}$. Poco después se diferenció del de identidad de género -la experiencia privada de pertenecer a uno $u$ otro sexo- acuñado por el psiquiatra y psicoanalista Robert Stoller (1968). Las normas de género dictan el comportamiento esperado según los roles de género y las virtudes y las caracteristicas de género que son rasgos psicológicos que se consideran masculinos o femeninos según hagan que quienes los posean se adecuen a las normas de género asignadas a hombres y mujeres (las características masculinas son virtudes en los varones y vicios en las mujeres y a la inversa). Y también tenemos el simbolismo de género que puede situar a los objetos inanimados y a los animales en un campo de representación de género, bien por asociación convencional, por proyección imaginativa o por pensamiento metafórico. Como es sabido, la sociologia ha convertido la categoría de género en uno de sus conceptos teóricos más influyentes ${ }^{32}$ que ha pasado a otras disciplinas y se ha convertido en fundamental a la hora de analizar la ciencia y la tecnología.

Los artículos que aparecen en este número utilizan, pues, como categoría de análisis la de género y una perspectiva claramente feminista que permite vre qué valores intervienen en nuestras teorias y prácticas científico-tecnológicas, a cuáles de ellos estamos ligados. El feminismo ha contribuido a desvelarlos, produciendo un cambio en la ciencia y en la tecnología, asi como en la idea que las personas tienen de ambas. Pero, ¿cómo? Elizabeth B. Silva se centra en la desestabilización que el feminismo ha producido en las "categorias y divisiones de varios campos del conocimiento", debido a que se ha dedicado a hacer visibles aspectos de la ciencia que antes no lo eran y a cuestionar qué y por qué se incluyen -o excluyen-ciertos aspectos del mundo.

Comienza presentando de forma breve cómo se han desarrollado lo que denomina "ciencias feministas" como parte de la política feminista y efectúa una serie de consideraciones acerca del papel de las distinciones locales del feminismo en el cambio de las disciplinas cientificas. En especial, y partiendo de la distinción ya formulada en los años setenta y ochenta entre feminismos liberales, socialistas y radicales, a pesar de las diferencias existentes entre ellos, establece la importancia de insistir en que las causas de opresión de las mujeres son sociales en sentido amplio (es decir, culturales, económicas y sociales) y no biológicas como se ha mantenido desde la antigüedad hasta nuestros dias (Amparo Gómez examina en su artículo algunos

ARBOR CLXXXI 716 NOVIEMBRE-DICIEMBRE (2005) 447-462 ISSN: 0210-1963 
aspectos de este uso de la biología para mantener la dominación económica y social de las mujeres). Pero ese consenso se rompe en los años noventa, con las acusaciones de etnocentrismo y racismo que efectúan las mujeres negras (fundamentalmente) y con la apreciación positiva de la diferencia, entendida no tanto de una manera biológica, como de la forma mediante la cual ulas culturas crean condiciones especificas para que ciertos grupos de mujeres o .mujeres individuales, vivan y se reproduzcan". De ese modo se cuestiona, a la vez, la distinción entre sexo y género, entre biologia y construcción social. Pero también se incide en la contextualidad y variabilidad de las mujeres, así como en el hecho de que para potenciar las capacidades y el poder de todas las mujeres hay que tener en cuenta las cualidades especificas de cada una de las mujeres.

Si se ha de tener en cuenta esas cualidades femeninas, habrá que intentar que aumente el número de mujeres estudiando y haciendo ciencia y tecnología. Ahora bien, ¿qué significa ese aumento, qué revela y qué esconde? Revela que hay diferencias entre hombres y mujeres, pero también entre las que cuentan o importan y las que no. Revela que las mujeres no avanzan en sus carreras a la misma velocidad que los hombres, sin que eso suponga inferioridad de resultados previos en las carreras de las mujeres. De hecho, algunos trabajos (Pérez Sedeño, 1996, Velho e Leon, 1998) muestran la enorme productividad de las mujeres en situaciones supuestamente improductivas (como en periodos de embarazos o partos) o situaciones supuestamente paradójicas, como el hecho de que en áreas donde las mujeres son minoritarias publican más que los hombres y a la inversa (donde son mayoria publican menos). La interpretación que hacen algunas estudiosas es que alli donde son mayoría, los hombres imponen su modelo de éxito. Pero, al margen de la generalidad de esos estudios o de la aceptación de esas interpretaciones, es posible pues, que las construcciones tradicionales de la ciencia esto es, como dice Elizabeth B. Silva, masculinas - sirvan a ciertos objetivos o fines que variarian si hubiera más mujeres en la ciencia. Por eso, las feministas han preparado una agenda para las ciencias y la tecnologia que incluye cuestiones sobre poder, sexualidad, exclusiones y refuerzo de las normas de género, entre otras cosas.

Como buena conocedora de las criticas formuladas en los años setenta y ochenta contra las feministas blancas norteamericanas y británicas fundamentalmente, y dado que toda agenda feminista se caracteriza por su carácter prác- tico, E. B. Silva se pregunta en qué medida esa caracteristicas generales expuestas y sus propuestas, son aplicables a Brasil, dada la multiplicidad de etnias, lugares y clases, que hace que las mujeres brasileñas vivan sus vidas privadas de manera muy diferente a como las viven las europeas o norteamericanas.

El trabajo de Patricia Tovar aborda las cuestiones de género en las ciencias sociales, en especial en antropologia. Su enfoque es especialmente interesante, pues combina el aspecto autobiográfico, la mirada de la antropóloga sobre si misma, junto con la reflexión crítica sobre su disciplina. 0 , mejor dicho, poniendo como pretexto diversas preguntas personales, se plantea cuestiones capitales en la antropología en particular y en las ciencias sociales en general -incluso en todas las ciencias-, añadiría yo.

Comienza Patricia Tovar efectuando un breve repaso del desarrollo de su disciplina desde comienzos del siglo veinte y de sus fundamentos, donde destaca que los principales investigadores fueran blancos, europeos o norteamericanos -con algunas mujeres destacadas- al servicio, por lo general, de los gobiernos occidentales o miembros de los cuerpos de paz y preocupados por "civilizar" las "sociedades primitivas" o exóticas (es decir, no occidentales) que estudiaban; dicho de otro modo, lo que trataban era de imponer su cultura y religión desde un marcado etnocentrismo.

Tras la Segunda Guerra Mundial y los cambios políticos que se producen en África, Asia y América, se comienzan a plantear preguntas que llevan entre otras cosas, a cuestionar algunos de los objetivos teóricos de la antropología, que eludían estudios de, y compromisos con, temas tales como la pobreza, la discriminación racial o la subordinación de la mujer. En el caso de Iberoamérica, la antropologia ha tenido un marcado carácter indigenista y enorme compromiso social que ha llevado a los antropólogos y antropólogas a militar en diversos movimientos armados, indigenistas, campesinos o democráticos. Pero, sobre todo, ya no se distingue entre "pueblos civilizados" y "primitivosi", con las connotaciones valorativas que esa diferenciación conllevaba, sino que se explora "al otro", para entendernos mejor, esto es, a todos, a ellos y a nosotros. ¿Y a nosotras?

Como ya señalé antes, lo singular del trabajo de Patricia Tovar es cómo su historia biográfica le sirve para reflexio-

ARBOR CLXXXI 716 NOVIEMBRE-DICIEMBRE (2005) 447-462 ISSN: 0210-1963 
nar sobre las mujeres en general, las antropólogas en particular y su disciplina. Las dificultades experimentadas como estudiante de post-grado en los Estados Unidos de América primero, como doctora e investigadora después, muestran de manera paradigmática y reflexiva las diferentes condiciones en que antropólogos de distinto sexo, pero también de diferente etnia, desarrollan su trabajo de campo, que es básico en la disciplina: en qué consiste este pilar fundamental de la antropologia, para qué se utiliza y cómo podría ser utilizada (en beneficio de qué y de quienes), en suma qué valores e intereses hay en juego.

El resultado del feminismo, es decir, de la consideración de que los valores contextuales forman parte consustancial en muchas disciplinas, es diverso: desde el cambio de definición de la disciplina -de "ciencia que estudia al hombre", con las connotaciones sabidas de este último término, a "ciencia de los seres humanos" - hasta la reevaluación de toda la disciplina a partir de los años setenta, gracias a la reconsideración del objeto de estudio. Pues, como sucede en otras disciplinas, el cambio de definición -o mejor, de caracterización- no es un simple empeño por cambiar un término por otro. Es cierto que las feministas que hablamos español sabemos que, en una de sus acepciones, "hombre" abarca a todo el género humano. Pero también sabemos que es doblemente genérico, pues las disciplinas que supuestamente se ocupaban de toda la especie humana tradicionalmente sólo se ocupaban de la mitad de la especie, del varón, de lo masculino ${ }^{33}$. El hecho de universalizar de verdad el objeto de estudio, es decir, de abarcar a hombres y mujeres, ha permitido muchas cosas, como, por ejemplo, cuestionar los aspectos teóricos de las desigualdades de género en las sociedades occidentales y su comparación con otras sociedades.

El trabajo de Amparo Gómez se ocupa de examinar la existencia y el papel de los valores en un caso concreto dentro de las disciplinas biomédicas del siglo diecinueve. En especial, analiza cómo los valores ideológicos androcéntricos dominantes intervinieron en los estudios del cerebro y de la teoría de la inferioridad mental de la mujer efectuados por P.J. Möbius a finales del siglo diecinueve.

Comienza A. Gómez con el análisis de los contenidos de la teoría de la evolución con respecto a las mujeres, en la versión más desarrollada al respecto, la De Herbert Spencer. En especial, la autora analiza los argumentos sobre la desigualdad física y mental de las mujeres con respecto a los hombres. Desigualdades y diferencias que vienen dadas, según los evolucionistas, por el papel o la función que la evolución ha asignado a las mujeres, la procreación, para lo cual no es necesario el desarrollo de cualidades superiores. Asi, además de la falacia lógica de deducir enunciados de "deber" de enunciados de "hecho" (esto es, pasar del enunciado que afirma que las mujeres son procreadoras a afirmar que no deben desarrollar otras cualidades) convierte Spencer una diferencia de ipso primero en diseño y luego en inferioridad. Una inferioridad intelectual inducida: las mujeres -afirma Spencer- podrian desarrollar su intelecto, pero eso iría en detrimento de su capacidad reproductora. De ese modo hace uso de una especie de principio de conservación de la masa y la energía "biológico-reproductivo", según el cual la energía empleada en el intelecto disminuiría la capacidad reproductora $y_{1}$ a su vez, el aumento de actividad cerebral aumentaría el volumen de ese órgano y disminuiría el de los ovarios.

Pero, seguramente, uno de los hechos que más influyeron en la construcción del discurso "cientifico" sobre la inferioridad mental de la mujer fueron los estudios del cerebro desarrollados desde la frenologia, la neurología y la antropología. Los frenólogos -guiados por los trabajos de J.F. Gall- mantenian (y explicaban) que existia una relación entre forma del cráneo y las caracteristicas mentales y conductuales de los individuos. Los antropólogos, por su parte, utilizaron los estudios frenológicos (en especial, la noción de indice cefálico) para fundamentar las caracteristicas mentales y comportamentales de los dos sexos, pero también de las razas y otros grupos humanos (como los obreros o los criminales).

Pero es en neurología, de la mano de los trabajos del autor que es objeto del trabajo de Amparo Gómez, P. J. Möbius, donde la tesis de la inferioridad mental de la mujer recibe mayor apoyo, hasta convertirse en piedra angular de la constitución del discurso científico sobre "la cuestión", como fue denominada en el siglo diecinueve. Basándose en estudios comparativos del fisiólogo y anatomista Rüdinger y $\sin$ cuestionar en absoluto su metodología ni su validez establece, tajantemente y de una vez por todas, el menor desarrollo de ciertas partes cerebrales de las mujeres y, por tanto, su inferioridad en todos los aspectos.

Amparo Gómez examina detenidamente los estudios y datos de Rüdinger empleados por Möbius y pone de manifiesto importantes anomalias metodológicas y argumenta-

ARBOR CLXXXI 716 NOVIEMBRE-DICIEMBRE (2005) 447\%462 ISSN: 0210-1963 
tivas, genéricamente sesgadas desde un principio. Por un lado, parte de la idea de que hombres y mujeres son diferentes y de la inferioridad de éstas, basándose en las afirmaciones de diversos autores $y$ en dichos populares, aunque desea confirmar esa idea basándose en las investigaciones neurológicas. Al margen del carácter no justificado del supuesto, obsérvese que digo "confirmar", no "contrastari. Cuando un investigador o investigadora propone una hipótesis, es decir, un enunciado que puede ser verdadero o falso, idea un procedimiento que le permita averiguar la verdad o falsedad de dicho enunciado. Poro cuando ya se dispone de una contrastación favorable a la hipótesis, aun asumiendo su verdad, se procede a contrastarla más veces y de diferentes maneras para corroborar su verdad. Por otro lado, al efectuar los estudios sobre cerebros, ya separados como masculinos y femeninos, y proceder a continuación a compararlos, se cae en una argumentación circular y falaz, en concreto, se comete la falacia de petitio principii: es decir, se está argumentando a partir de premisas (experimentales en este caso) entre las cuales se incluye la diferencia entre cerebros de hombres y mujeres, a la conclusión de que los cerebros de hombres y mujeres son diferentes. Pero, además, entre las premisas tenemos al menos una sin justificar, a saber, que esa diferencia es inferioridad. Eso hace que la argumentación no pueda justificar la conclusión a la vez que, como esa premisa pertenece al conjunto de las creencias de base o de partida, sesga cualquier observación y conclusión.

El artículo de Carmen Mataix se construye alrededor de un libro que tuvo un éxito editorial inusual en la sociedad occidental actual, a pesar del tema de que trataba, la filosofía en su historia tal y como aparece en El mundo de Sofía de J. Gaardner. Según Carmen Mataix, este libro, publicado a finales del siglo veinte, se encuandra en ese género literario típico de la Revolución Científica y de la Ilustración que se dio en llamar "literatura científica para damas'. En efecto, la nueva ciencia y la nueva filosofía pusieron al alcance de la clase alta europea los nuevos instrumentos, abundaban las exhibiciones científicas, no faltaban gabinetes de ciencias -fisicos, de historia natural, etc.- en las mejores casas. Así que comenzaron a aparecer toda una serie de libros de divulgación científica. Y como no era de buen tono que una dama fuera completamente ignorante de las modernas teorias (siempre que no pretendiera dedicarse profesionalmente a las ciencias duras 0 mucho menos a la medicina), comenzaron a proliferar libros especialmente escritos para las damas. Benjamin
Martin, por ejemplo, escribio The Young Gentleman and Lady's Philosophy, donde manifestaba que saber ciencia era "una gracia peculiar en el bello sexo»34.

La aceptación de las teorias de Newton en todo el continente europeo (gracias, entre otras cosas, a la excelente traducción al francés que hizo Madame de Chatellet) tuvo la consecuencia de que hubo que rescribir las popularizaciones de mecánica, adoptando la mayoria el formato de esa literatura para damas. $Y$ así, surgen toda una serie de libros, de los cuales, sin duda uno de los más famosos es II newtonianismo per le dame, de Francesco Algeroti (publicado en 1737), en el que se expone la óptica y física newtoniana. Pero no hay que dejarse engañar por el género al que se adscriben estas obras, pues no todas las popularizaciones dirigidas a las señoritas eran de hecho divulgaciones. Por ejemplo, Charles Leadbetter dedicó su Astronomy: or the True System of the Planets Demonstrated (1727) a Mrs. Catherine Edwin quien según afirma el autor tenia gran erudición y habilidad en Ciencias matemáticas, en especial en las celestes; y así debia ser, a juzgar por la obra, plena de tablas astronómicas, complejos cálculos matemáticos y toda una serie de tecnicismos, mucho más de los usuales en los otros libros "para damas». Las popularizaciones cientificas fueron de gran importancia. Por ejemplo, las teorias mecanicistas de Descartes fueron dadas a conocer a un público más amplio gracias a La pluralidad de los mundos de Bernard Le Bovier de Fontenelle (1657-1757): en cinco tardes consecutivas, la marquesa de G., a quien está dedicada la obra, es instruida en el complejo universo copernicano y cartesiano ${ }^{35}$... condescendientemente. La obra de Fontenelle tuvo mucho éxito, (entre otras cosas, por que hablaba de la posibilidad de extraterrestres), y fue traducida al inglés por Aphra Behn (1640-1689). Esta versátil autora, viajera y aventurera, a pesar de no ser una "experta" en astronomia encontró en dicha obra tantos fallos y la halló tan susceptible de críticas que decidió escribir sus propias ideas al respecto: "Debo decirte sinceramente que [Fontenelle] ha fracasado en su proyecto; pues al pretender hacer familiar esta parte de la Filosofia Natural, ha caido en el ridículo ... Y por lo que se refiere a su Lady Marquiese, le hace decir muchas tonterías, aunque a veces hace observaciones tan inteligentes y eruditas que los mejores filósofos de Europa no las podrian hacer mejorn ${ }^{36}$.

Pues bien, Carmen Mataix señala la semejanza existente entre la obra de Fontenelle y la de Gaardner, salvando las

ARBOR CLXXXI 716 NOVIEMBRE-DICIEMBRE (2005) 447-462 ISSN: 0210-1963 
distancias que los siglos imponen entre ambos. Lo que más sorprende en realidad es lo poco que han variado los estereotipos sobre las mujeres en estos siglos: un libro escrito por un nórdico y a finales del siglo veinte sigue mostrando las mismas cotas de actitudes paternalistas, moralistas y desigualdades jerárquicas. La destinataria, en el caso de la obra de Gaardner, tiene que ser necesariamente una adolescente, pues terminado el siglo pasado, si hubiera sido una mujer adulta, el autòr no habria salido indemne. Pero la propia elección de una adolescente y no de un adolescente, le delata, indica la apreciación real que tiene el autor de las mujeres: eternas adolescentes, o su deseo de que así sean. El trabajo de Carmen Mataix es una muestra clara de lo que supone hacer crítica desde la perspectiva feminista y de género: estoy completamente segura de que quien lea El mundo de Sofía después de leer este articulo, considerará que tiene entre sus manos un libro muy distinto.

El artículo de Marta González es una excelente contribución al análisis de las relaciones entre ciencia, tecnologia y sociedad, centrada en la consideración de cómo factores sociales afectan los contenidos y prácticas científico-tecnológicas de la sexologia. En el trabajo se defiende la participación de valores contextuales en el modelado de contenidos y prácticas científicas a través de decisiones relativas a los problemas, métodos y fines de la investigación. Estas decisiones, normalmente adscritas al "contexto de descubrimiento" y consideradas irrelevantes para la evaluación de los productos científicos, muestran ser, por el contrario, centrales para los procesos de justificación, ya que los criterios de aceptación o rechazo son en cierta medida dependientes de prioridades epistémicas mediadas por valores contextuales. Defender la participación de valores contextuales es un paso previo para defender la participación de valores de género en determinados episodios cientificos. Por eso se ha escogido para ilustrar la tesis defendida el caso de estudio de la sexologia, una disciplina que se encuentra en proceso de negociar lo que pueden considerarse formulaciones de problemas, prácticas y teorias propias; y donde la evaluación de sus procesos y productos depende de valores contextuales relativos a fines, expectativas y preconcepciones, en muchos casos relacionados con el género. Analizando el desarrollo de la sexologia desde sus origenes en la Alemania del siglo XIX hasta las criticas feministas al paradigma impuesto por Masters y Johnson se mostrará el modo en que valores contextuales marcan las prioridades epistémicas estable- ciendo asi los criterios de aceptación o rechazo de teorias y prácticas.

El trabajo de Diana H. Maffía plantea algunos de los problemas más importantes y menos tratados en filosofía en general y en teoria del conocimiento en particular. Presenta dos aspectos importantes: la tensión entre conocimiento y emoción (por lo general resuelta a favor de la primera parte del par) y cómo conocemos las emociones propias y las ajenas. Dada la importancia de este estudio para mostrar nuestra vinculación con el mundo y con los otros, es realmente insólito que las emociones hayan recibido tan poca atención por parte de la filosofia de la ciencia y de las ciencias cognitivas.

En la filosofía contemporánea, las emociones se utilizan como forma de acotar las posibles alternativas en la elección racional de los denominados "sistemas expertos", pareciendo, pues, que surge una paradoja: el conocimiento se opone a emoción (junto con otras dicotomias excluyentes y jerárquicas como objetivo/subjetivo, activo/pasivo) pero se utilizan las emociones para comprender la racionalidad. Las emociones permiten enmarcar nuestras decisiones definiendo los parámetros a tomar en cuenta en evaluaciones o decisiones concretas y entresacando sólo algunas de las alternativas posibles en el proceso de deliberación racional. Es decir, "las emociones... serian importantes para la racionalidad porque rebajan a una medida manejable el número de consideraciones relevantes para la deliberación racional y proporciona el marco indispensable sin el cual la cuestión de la racionalidad ni siquiera podría surgirn.

También los estudios feministas han llamado la atención sobre la evaluación de la corporalidad y la emocionalidad con respecto a la filosofía y la ciencia, en especial al considerar que las caracteristicas masculinas son las humanas, es decir, las de todos los seres humanos, lo que ha llevado a una normatividad científico-filosófica no universal y espuria. Al considerar esta nueva tradición, Maffía abre el camino a la discusión acerca de cómo conocemos las emociones. Tras hacer un repaso a la reciente tradición cognitivista, reconoce que el estudio de las emociones es uno de los grandes retos de las ciencias cognitivas (en especial de la psicología cognitiva), pero, en su opinión, comete un gran fallo en el mismo punto de partida: la metáfora de la mente/ordenador, que es una metáfora informática del conocimiento, es decir, como procesamiento de informa- 
ción; de ese modo, se ocupa sólo de una de las direcciones posibles del camino, la que va de la cognición a la emoción, pero no a la inversa.

Aún así, la autora reconoce el interés del proyecto cognitivo que se caracteriza por pretender determinar algún tipo de estructura general en las situaciones posibles que desencadenan las emociones y especificar su estructura psicológica. Aprecia positivamente el intento de describir cómo se evalúan cognitivamente las emociones, cómo se organizan y los procesos cognitivos implicados, es decir, el interés por descubrir lo que podriamos denominar una "gramática de las emociones", es decir, la explicitación de los principios implicitos que se incorporan en la experiencia emocional normal.

Para ello, se propone una tipología de las emociones (en lugar de ocuparse de los distintos estados emocionales) y el estudio de cómo se relacionan entre sí. Dicha tipología parte de la idea básica de que los aspectos principales que hay que tener en cuenta sobre cómo percibimos el mundo son tres: acontecimientos, agentes y objetos. Las emociones son "reacciones con valencia" a algunos de estos aspectos, que dan lugar a tres tipos básicos de emoción: estar contento (frente a estar disgustado), aprobación (frente a desaprobación) y agrado (frente a desagrado).

También examina la autora el programa de H. Maturana, que es bien distinto. Para comenzar, su análisis se enmarca en la biología del conocimiento, pero considera que no hay que contemplar la racionalidad como característica de lo humano, pues eso relega la emocionalidad al aspecto animal. Y lo que sucede es que todo sistema racional tiene fundamento emocional, que es su condición de posibilidad: cuando estamos bajo cierta emoción, hacemos ciertas cosas, aceptamos ciertas argumentaciones, diferentes a las que hacemos o aceptamos bajo otras emociones. Las emociones están vinculadas con lo social y el lenguaje, con lo típicamente humano. $Y$, como afirma Mafia, reflexionar sobre la filosofía y la ciencia desde esta perspectiva puede ser escalofriante. Porque a la ciencia y a la filosofía se les ha hurtado históricamente las emociones y eso conlleva fallos cognitivos y morales notables, no es algo ensalzable y que hay que perseguir, como se nos ha hecho creer.

El trabajo de Ana Sánchez se enmarca en lo que se denomina epistemología de la complejidad del pensador francés Edgar Morin, los estudios CTS (de ciencia, tecnologia y sociedad) y la teoria feminista. Utiliza este marco complejo para analizar una de las tecnologías más impactantes y controvertidas por los múltiples aspectos implicados, del momento: las tecnologias de reproducción asistida. Frente a la idea tradicional de separar la ciencia y la tecnología de sus usos y consecuencias, la autora presenta su análisis como una forma de poner de manifiesto que hay que analizar y evaluar nuestras tecnologias de modo que se puedan efectuar reclamaciones acerca de su concepción, implementación y uso y evitar consecuencias indeseadas.

Para ello, examina el discurso biomédico a través de las metáforas utilizadas, que ayudan a identificar o desvelar los supuestos, creencias y valores que guian la metodología y prácticas biomédicas. Las metáforas usadas remiten a dos discursos y dos sistemas sociosimbólicos que modelizan y fragmentan el cuerpo y fijan el papel de la mujer como madre, lo que produce un círculo vicioso de necesidades/ofertas/necesidades de que no pueden escapar las mujeres.

Pero los valores del orden patriarcal no se encuentran sólo en el discurso biomédico; el discurso jurídico y el usado en los medios de comunicación comparten los mismos valores y claves, lo que impide una información critica en los medios de comunicación, o una legislación que no vaya a la zaga de la tecnociencia. La omnipresencia de estos valores queda expresamente manifestada en lo que Ana Sánchez denomina la "metáfora de la culpa" que tiene consecuencias importantes por lo que se refiere a las tecnologias terapéuticas y a la evaluación de riesgos y resultados. Por todos esos motivos, la autora, al igual que otras voces feministas, aboga pr un enfoque plural que refleje las distintas voces y razones, los diferentes intereses y discursos, en fin, las relaciones de poder y los valores existentes de manera más o menos explícita o implícita.

En "Conocimientos de lo cotidiano: situaciones e historias" Elizabeth B. Silva pretende discutir los antecedentes y consecuencias de las construcciones de nuestras categorías para el conocimiento, en la vida cotidiana y en la práctica científica. Como el resto de los artículos que aparecen en este volumen, mantiene la idea general de que la elección de lo que es importante en el campo del conocimiento no está libre de valores contextuales, no es el resultado sólo de criterios metodológicos y científicos en la acepción tradicional mencionada al comienzo. Para mostrarlo, comienza examinando la tensión existente entre la metodología 
cuantitativa y la cualitativa como metodologias convencionales para obtener conocimiento.

Aunque mantiene que en el área de ciencias sociales y en la de los estudios feministas se acepta que los factores externos, incluida la ideología de género, conforman el conocimiento, existen tensiones acerca de cómo nuestros valores, creencias e ideología afectan el conocimiento, lo que supone un desafio radical a las formas tradicionales de hacer ciencia. En especial, y como ya subrayaban los primeros estudios feministas, las voces de las mujeres estaban ausentes, disminuidas, distorsionadas o eran inapropiadas. $Y$ al hacer hincapié en el carácter cuantitativamente minoritario de las mujeres en la ciencia, se contraponia la alternancia cualitativa. Pero esa oposición cuantitativo/cualitativo no es sólo una polémica sobre metodologías, sino sobre procesos culturales muy profundos, sobre concepciones diferentes acerca de qué es el conocimiento. Una de esas concepciones parte de la idea de que el conocimiento está situado, en algún lugar, tiempo, cultura o cuerpo. Esos conocimientos situados son parciales, inacabados y contingentes. Por eso, sólo la suma de esos conocimientos permite una perspectiva "privilegiada" de conocimiento.

Esta concepción del conocimiento como conocimiento situado lleva a la autora a reflexionar sobre las implicaciones que la vida cotidiana de las mujeres, las historias de lo cotidiano, tienen para las maneras de conocer. Las historias de las mujeres nos hablan de relaciones tradicionales de género que refieren a determinados roles - por ejemplo-, en el trabajo de cuidar los cuerpos de los hombres, los niños, los enfermos, los propios cuerpos de las mujeres, pero también de los locales donde viven esos cuerpos. Ahora bien, las prácticas institucionales, tecnológicas, politicas y económicas refuerzan esos roles de modo que no se considera, por lo general, que las actividades de las mujeres forman parte de la historia y la cultura humana, debido a su invisibilidad ${ }^{37}$. Pero no sólo son invisibles las actividades de las mujeres para los hombres, en realidad, para toda la sociedad. Existen muchas mujeres cuyas vidas, cuyas historias, son invisibles para las pocas mujeres que tienen poder y a las que ayudan cuidando a sus hijos, sus compañeros, los lugares donde habitan, donde trabajan. Por eso, hay que dar cuenta del punto de vista de todos y todas las excluidas.

El último trabajo, realizado por Sara Rietti y Diana H. Maffia, aborda las desigualdades de género, en especial la escasa pre- sencia de mujeres en las denominadas "ciencias duras", esto es en matemáticas, física, química e ingenierias, y en puestos de responsabilidad, no sólo en esas áreas sino también en politica. Pero abordan ese problema desde una perspectiva diferente, pues intentan mostrar un paralelismo importante entre el ejercicio de la ciencia y el de la ciudadanía. Diferente porque, aunque comparten con el resto de los trabajos la asunción del modelo androcéntrico en que se encuadran la ciencia y la política, vinculan las dos situaciones: la de las mujeres en el sistema científico-tecnológico y en el sistema político.

La tesis que pretenden mostrar a lo largo del trabajo es que, para explicar la situación de las mujeres tanto en ciencia como en política, no basta la explicación usual que responsabiliza al estereotipo de género y a la cultura androcéntrica y patriarcal. Esos factores son importantes, pero no los únicos.

Señalan las autoras la frecuencia con que las mujeres de las disciplinas antes mencionadas o con cargos políticos destacados afirman no haberse sentido discriminadas por razón de su sexo y explican las renuncias, paradas y estancamientos en sus carreras como decisiones personales, tomadas, por lo general, para evitar conflictos que suelen darse entre la esfera pública/profesional y la privada. El problema, según las autoras, es que las habilidades en que se socializa a las mujeres son contrarias a las que se les exige en la vida profesional, lo que supone el ejercicio de la violencia sobre las capacidades y hábitos aprendidos para la esfera privada, pero que se consideran no sólo inadecuados, sino nocivos, para la esfera pública, en este caso, la ciencia y la tecnología, así como la politica.

La pregunta que se plantean las autoras es qué sucedería, tanto en ciencia como en política, si, como resultado del aumento de mujeres, cambiaran las normas. Qué sucedería si, en lugar de explicar la ausencia (o deserción) de las mujeres de ciertas áreas por su incapacidad de adecuarse al proyecto que conforman, consideráramos que tienen razones fundamentales para obrar así. Qué pasaría si se asignara valor cognitivo positivo a las capacidades supuestamente típicas de las mujeres. En un difícil ejercicio epistémico crítico para evitar caer en la idealización esencialista, las autoras abogan por la valoración epistémica de cualidades que no se suelen considerar positivas para la construcción del conocimiento. Peor, como ellas mismas dicen, no se trata tan sólo de un ejercicio de imaginación, pues ya hay muchos ejemplos (y creo que este libro es uno de ellos).

ARBOR CLXXXI 716 NOVIEMBRE-DICIEMBRE (2005) 447-462 ISSN: 0210-1963 
El ejercicio de la ciencia y de la ciudadania presenta aspectos significativamente semejantes o paralelos. No sólo por el hecho de que la ciencia moderna aparezca en Europa como proyecto a la vez que se diseña el Estado Moderno. No sólo porque tanto en el proyecto científico como en el político esté implícito un modelo de sujeto neutro, no corpóreo y separado del objeto. No sólo porque en política -como en ciencia- se prima la lealtad y la obediencia, en vez de la originalidad, la novedad y modelos alternativos.
Como manifiestan las autoras «no es aventurado... ॥. El impacto que puede suponer tan sólo el hecho de asumir una "autoridad perceptivan distinta a la que se mantiene en política, ya sería enorme, pues de ese modo se enriquecerian la ciencia y la política, porque ninguna es exclusiva de un pais, o de un grupo, sino de toda la sociedad. Todos los ejemplos, como se ve muestran la pertinencia de estudiar el influjo del género en nuestras teorias y prácticas tecnocientífica.

\section{NOTAS}

1 La expresión fue acunada por P. Kitcher (1993), quien señaló que era la "concepción de la ciencia con mayor anuencia científica y popular» (op. cit. p. 3).

2 Lacey (1999)

3 Véase, por ejemplo, M. Masterman (1970), donde identifica por lo menos veintiún usos distintos del término.

4 Kuhn, 1962, p. 182.

5 "Yo describiria esos compromisos como creencias en modelos concretos y ampliaría la categoría de "modelos" de modo que incluyera también la variedad relativamente heuristican. Op. Cit. p. 184.

6 "... en principio, las soluciones a problemas concretos que los estudiantes encuentran desde el comienzo de su educación científica, sea en los laboratorios, los exámenes, o al final de los capitulos en los libros de texton (pp. 186-7).

7 Kuhn, op. cit., pp. 184-85. Las cursivas son mías.

8 En realidad el artículo corresponde a la Manchette Conference, pronunciada en la Universidad de Furman el 30 de noviembre de 1973

9 Kuhn, 1977, p. 346

10 Op. Cit., p. 348.

11 El ejemplo que pone aqui Kuhn es el neoplatonismo de Kepler.

12 Véase, por ejemplo Giere (1991) o Diez Calzada y Moulines (1997).

13 Kuhn, 1977, p. 351.

14 Kuhn, 1977, p. 352. Sobre los olvidos o dobles raseros, tanto por parte de la concepción heredada como de los filósofos historicistas -no sólo Kuhn-, sino también Lakatos y Laudan, véase Fuller, 2000, pp. 82-92.

15 Kuhn, 1977, pp. 354-55.

16 lbidem.

$460 \quad 17$ Merton (1973)
18 Las criticas a las normas mertonianas han sido muchas. Algunos sociólogos de la ciencia se han basado en su incumplimiento para despojar de toda validez a la ciencia. Una de las evaluaciones más equilibradas se puede encontrar en ZIMAN 2000, capítulos. 3, 5, 6 y 7.

19 Hay determinadas estructuras sociales que contribuyen a reforzar esos mecanismos, como ciertos sistemas de recompensa tales como los premios, la eponimia (la asignación del nombre del «descubridorn a un descubrimiento, ley, etc.) 0 los protocolos de publicación.

20 El País, 23-III-2006.

21 Véase, por ejemplo, Sheldon et al. 1989, Allchin (1998) y Orlans, 1993.

22 Según el informe oficial, Chen robó la tecnologia de otras compañias (una de ellas, supuestamente Motorota) para fabricar sus chips, usados para procesamiento digital de señales en productos como reproductores de MP3 y teléfonos móviles. Aunque estos son casos famosos recientes los denominados "fraudes científicos" abarcan todas las épocas, paises y disciplinas. Véase, por ejemplo, Broad y Wade (1983), Di Trocchio (1993) o Freeland Judson (2004).

23 Allchin, pp. 3.

24 De hecho, muchos autores hablan de "tecnociencia" (Echeverría, 2002).

ARBOR CLXXXI 716 NOVIEMBRE-DICIEMBRE (2005) 447-462 ISSN: 0210-1963 
25 En mi opinión, el debate acerca de la obtención de células troncales 0 umadren a partir de embriones humanos no se ha presentado adecuadamente en algunos medios de comunicación. Por un lado se presenta como un conflicto entre quienes quieren que la ciencia «avance» apelando a supuestos criterios científicos de búsqueda de conocimiento o de la verdad y de aplicabilidad para curar enfermedades y quienes consideran inaceptable tal investigación por razones uideológicas", en concreto religiosas. Pero, los valores subyacentes en ambos casos son sociales o éticos, basándose cada parte en distintas ideologias: unos mantienen que no es correcta la investigación porque los embriones son "personas" o pueden llegar a serlo, los otros dicen que eso no es así (y apelan además a una cierta «ética del despilfarro»: dado que los embriones no se pueden implantar ya, se desperdiciarian). Los valores presentes en esta disputa ocultan otros: por ejemplo, la ocultación de valores de género (o más bien, la presencia de valores sexistas) esconde la procedencia de los embriones y la necesidad para su obtención de contar con miles de mujeres que se sometan a las técnicas de reproducción asistida (en especial estimulaciones ováricas y laparoscopias) cuyas consecuencias no están bien estudiadas ni determinadas, pero, en principio, potencialmente peligrosas. $Y$ como las mujeres, una vez más, son borradas como objeto/sujeto de la experimentación (obsérvese como en las representaciones gráficas de la obtención de las "células madre", a pesar del nombre no aparecen ovarios de mujeres, de los que se obtienen los óvulos) también desaparecen otras posibilidades de obtención de dichas células, como la médula ósea. Si se acepta la investigación con embriones congelados, ¿qué sucederá cuando se termine el "stock" existente? ¿No se da por sentado que seguirá habiendo, porque las mujeres seguirán sometiéndose a las TRA y produciéndose "excedentes»? Por supuesto, mis valores son distintos a los de las partes implicadas en la disputa, pero resulta cuando menos sorprendente, que no aparezcan estas consideraciones en el debate que nos presentan los medios de comunicación.
26 Takacs, 1996.

27 Véase Hager (1997) o Schiebinger (1999). 28 Hardin, 1968, pp. 1243.

29 Ciertamente, no por lo que respecta a algunos valores, que deberán ser "acordados" para dar cierta cohesión social, de manera más o menos clara.

30 Pérez Sedeño (2005)

$31 \mathrm{El}$ concepto en cuestión surgió de su experiencia "en el tratamiento de casos donde no se producia la "normal" convergencia entre el sexo biológico, el sexo psicológico y el deseo heterosexual" (Garcia Dauder, en prensa).

32 (Oakley, 1972)

33 Véase, por ejemplo, Pérez Sedeño (2000) y (2002).

34 Escrita en forma de diálogo entre un hermano y una hermana, esta última manifestaba: «A menudo deseo que no resulte $\tan$ masculino que una mujer hable de Filosofía en Compañía... ?cuán feliz será la época en que las Damas puedan pretender modestamente poseer conocimientos y parezcan entendidas sin Singularidad y Afectación!n. Citado en Pérez Sedeño (1992).

35 En él la Tierra se halla firmemente apoyada en un vértice, que es el que gira alrededor del Sol.

36 A. Behn, An Essay on Translation and Translated Prose.

37 Por eso, una parte del trabajo feminista en ciencia ha sido la recuperación de las figuras femeninas y sus aportaciones.

\section{REFERENCIAS BIBLIOGRÁFICAS}

Allchin, D. (1998): "Values in Science and in Science Education», en "B. J. Frase y K.G. TOBIN (eds.)" International Handbook of Science Education, Dordrecht, Kluwer Academic Publishers.

Broad, Willam y Wade, Nicholas (1983): Betrayers of the Truth, Simon \& Schuster.

Di Trocchio, Federico (1993): Le bugie della scienza. Perche» e come gli scienziati imbrogliano, Milán, Mondadori (trad. Esp. En Alianza Ed. 1995).

Díez Calzada, José Antonio y Moulines, Ulises (1997): Fundamentos de Filosofía de la Ciencia, Barcelona, Ariel.

Echeverria, Javier (2002): Ciencia y valores, Barcelona, Destino.

Fuller, S. (2000): Thomas Kuhn. A Philosophical History for Our Times, Chicago-Londres, The University of Chicago Press.

Garcia Dauder, Silva (en prensa): «Ingenieria bioconductual al servicio de la normalización: Vigilando las fronteras del sexo", en En Romero Cuadra, José Luis y Álvaro Vázquez, Rafael (ed.), Antipsychologicum. Barcelona, Ed. Virus.

Giere, Ronald (1991): Understanding Scientific Reasoning, Nueva York, Holt, Reinhart and Winston.

Hager, Lori, ed. (1997): Women in Human Evolution, Nueva York, Routledge.

Hardin, Garret (1968): "The Tragedy of the Commons", Science, 162, pp. 12431248.

Judson, Horace Freeland (2004): The Great Betrayal : Fraud in Science, Hardcourt Books.

Kitcher, Phillip (1993): The Advancement of Science. Science without Legend Objectivity without Illusions, Nueva York-Ixford, Oxford University Press. (Hay trad. esp. de H. Islas y L. Manriquez con revisión técnica de Carlos López Beltrán.

Kuhn, Thomas S. (1962): The Structure of Scientific Revolutions, Chicago, 1962. Trad. esp. La estructura de las revoluciones cientificas, México, F.C.E., 1968.

- (1977): "Objectivity, Values and Theory Choicen, en The Essential Tension, Chicago, The Chicago Univ. Press. Trad. Cast. La tensión esencial, México, F.C.E. 1982. 
Lacey Hugh (1999): Is Science value free? Values and scientific understanding, Londres-Nueva York, Routledge.

Masterman, Margaret (1979): "The Nature of a Paradigm" en Lakatos, I. Y Musgrave, A. (eds.), Criticis, and the Growth of Knowledge, cambridge University Press. Hay trad. Esp. De F. Hernán con Introducción de Javier Muguerza, La crítica y el desarrollo del conocimiento, Barcelona, Grijalbo, 1975

Merton, Robert K. (1973): The sociology of Science, Chicago, Universty of Chicago Press (trad, esp. en Alianza Ed. 1977).

Money, John, Hampson, J.L. y Hampson, J.G. (1955): Hermaphroditism:

Recommendations concerning assignment of sex, change of sex, and psychologic management. Bulletin of the John Hopkins Hospital, 97: 284-300.

Oakley, Ann (1972): Sex, Gender and Society. Revised Edition. Arena y Gower Publishing, Hampshire.

Orlans, B. (1993): In the Name of Science: Issues in Responisible Animal Experimentation, Oxford, Oxford University Press.

Pérez Sedeño, Eulalia (1992); «Mujer, ciencia e llustraciónn, en Celia Amorós (ed.) Feminismo e llustración, Ediciones de la CAM/Instituto de la Mujer.

- (1996): «Family versus career in women mathematicians", en Women and Mathematics, Copenhague, European Women in Mathematics Society.

- (2000): "Institucionalización de la ciencia y valores contextuales: un caso ejemplar», cadernos Pagú, vol. 15, pp. 77-102.

- (2002): «Ciencia y Filosofía: Una nueva miradan, Clepsydra, 1, pp. 13-30.

- (2005): "La percepción de la ciencia y la tecnologia de "la otra mitad" ", en La percepción pública de la ciencia en España: La encuesta de 2004, Madrid, FECYT.

Sheldon, M., Whiteley, W.P., Folker, B. Hafner, A.W. y Gaylin, W. (1989): «Nazi Data: Dissociation of Evil. Commentary", Hastings Center Report, 19 (4), 16-18.

Schiebinger, Londa (1999): Has Feminism changed science? Harvard University Press.
Stoller, Robert J. (1968). Sex and Gender. The development of masculinity and femininity. New York: Science House.

Takacs, D. (1996): The Idea of Biodiversity; Philosophies of Paradise, Baltimore, Johns Hopkins University Press.

Velho, Lea y Leon, Elena (1998) «A construcao social da producao cientifica por mulheres", in Cadernos Pagu 10: 309344.

Ziman, John (2000): Real Science, Cambridge, CUP. Trad. Esp. ¿Qué es la ciencia?, Madrid, CUP.

ARBOR CLXXXI 716 NOVIEMBRE-DICIEMBRE (2005) 447-462 ISSN: 0210-1963 